\title{
Iterated rings of bounded elements and generalizations of Schmüdgen's Positivstellensatz *
}

\author{
Markus Schweighofer
}

\begin{abstract}
Let $A$ be a commutative $\mathbb{R}$-algebra of finite transcendence degree $d \in$ $\mathbb{N}$. We investigate the relationship between the subring of (geometrically) bounded elements

$$
H(A):=\{a \in A|\exists \nu \in \mathbb{N}:| a \mid \leq \nu \text { on Sper } A\}
$$

and the subring of arithmetically bounded elements

$$
H^{\prime}(A):=\{a \in A \mid \exists \nu \in \mathbb{N}: \nu+a \text { and } \nu-a \text { are sums of squares in } A\} .
$$

Obviously, $H^{\prime}(A) \subseteq H(A)$. In 1991, Schmüdgen proved the remarkable theorem that $A=H(A)$ implies $A=H^{\prime}(A)$ if $A$ is finitely generated. In 1996, Becker and Powers considered the chain $A \supseteq H(A) \supseteq H(H(A))=$ : $H^{2}(A) \supseteq \ldots$ and showed $H^{d}(A)=H^{d+1}(A)$. In 1998, Monnier related both results and conjectured $H^{d}(A)=H^{\prime}(A)$ which generalizes both of them at the same time. We prove this conjecture and develop tools to study $H^{\prime}(A)$. One of the applications is the following: If $a \in A$ is "small at infinity" and $a \geq 0$ on Sper $A$, then $a+\varepsilon$ is a sum of squares in $A$ for every $\varepsilon>0$.
\end{abstract}

\section{Introduction}

In this paper, all rings are tacitly assumed to be commutative and to have a unit element. A subset of a ring is called a preordering if it contains all squares and is closed under addition and multiplication. We call a preordering proper if it does not contain -1 . For instance, in any ring, the preordering generated by elements $t_{1}, \ldots, t_{m}$ (i.e., the smallest preordering containing $t_{1}, \ldots, t_{m}$ ) consists of all elements of the form

$$
\sum_{e \in\{0,1\}^{m}} \sigma_{e} t_{1}^{e_{1}} \cdots t_{m}^{e_{m}}
$$

where every $\sigma_{e}$ is a sum of squares. In 1991, Schmüdgen proved by functional analytic methods the following Positivstellensatz (which can easily be derived from several results which we will prove, for instance, from Theorem 5.1 by setting $g=1)$ :

${ }^{*} 2000$ Mathematics Subject Classification: 13J30, 14P10, 11E25, 44A60 
Theorem 1.1 (Schmüdgen) Let $t_{1}, \ldots, t_{m} \in \mathbb{R}\left[X_{1}, \ldots, X_{n}\right]$ be polynomials. Let $T$ be the preordering generated by $t_{1}, \ldots, t_{m}$ in the polynomial ring and

$$
S=\left\{x \in \mathbb{R}^{n} \mid t_{1}(x) \geq 0, \ldots, t_{m}(x) \geq 0\right\} .
$$

If $S$ is bounded, then for every polynomial $f \in \mathbb{R}\left[X_{1}, \ldots, X_{n}\right]$ we have

$$
f>0 \text { on } S \Longrightarrow f \in T \text {. }
$$

Note that clearly $f \in T$ implies $f \geq 0$ on $S$, but the converse does not hold, see [Ste].

In 1998, Wörmann published an algebraic proof of Schmüdgen's theorem which revealed that its really new content deals rather with boundedness than with positivity $[\mathrm{BW}]$. To explain this, we have to introduce some terminology.

A preordered ring is a ring together with a fixed preordering. We will use the same symbol $T$ to denote the fixed preordering of any preordered ring. This should not bring difficulties, like using + to denote the addition in any ring does not. In case of doubt, we will write $T_{A}$ for the fixed preordering of the preordered ring $A$.

\section{For the rest of the introduction let $A$ and $B$ be preordered rings.}

We say that $B$ is a preordered subring of $A$ if $B$ is a subring of $A$ endowed with the preordering $T_{A} \cap B$.

We write Sper $A$ for the real spectrum of $A$, i.e., for the space of all orderings of the ring underlying $A$ which contain the fixed preordering $T$ of $A$. We will make extensive use of the real spectrum Sper $A$ and the usual spectrum $\operatorname{Spec} A$ where the latter depends only on the ring structure of $A$. On each of these sets we will consider the usual topology (if nothing else is said) as well as the constructible topology.

Definition 1.2 We call the preordered subrings

$$
\begin{aligned}
H(A) & :=\{a \in A \mid \exists \nu \in \mathbb{N}: \nu \pm a \geq 0 \text { on Sper } A\} \quad \text { and } \\
H^{\prime}(A) & :=\{a \in A \mid \exists \nu \in \mathbb{N}: \nu \pm a \in T\}
\end{aligned}
$$

of $A$ the ring of (geometrically) bounded elements and the ring of arithmetically bounded elements, respectively.

To see that $H^{\prime}(A)$ is indeed closed under multiplication, use the identity

$$
3 \nu^{2} \pm a b=(\nu+a)(\nu \pm b)+\nu(\nu-a)+\nu(\nu \mp b) .
$$

Obviously, $H^{\prime}(A) \subseteq H(A)$ always holds.

Now consider the situation in Schmüdgen's theorem 1.1 and let $A$ be the polynomial ring $\mathbb{R}\left[X_{1}, \ldots, X_{n}\right]$ equipped with the preordering $T$ generated by $t_{1}, \ldots, t_{m}$. Then $S$ embeds densely in Sper $A$ and therefore

$$
H(A)=\left\{f \in \mathbb{R}\left[X_{1}, \ldots, X_{n}\right] \mid f \text { is bounded on } S\right\} .
$$


For that reason, the closed set $S$ is bounded if and only if $A=H(A)$. Hence it is a consequence of Schmüdgen's theorem that for an $A$ of this particular form

$$
A=H(A) \Longrightarrow A=H^{\prime}(A)
$$

In the first step of his proof of Theorem 1.1, Wörmann showed this implication. Actually, his proof works for any finitely generated (in the following often abbreviated by $f . g$.) $\mathbb{R}$-algebra $A$ equipped with an arbitrary (not necessarily f.g.) preordering. In a second step, he applied the following old theorem which is usually attributed to Kadison and Dubois while it was recently revealed that it might more likely be due to Krivine or Stone. We include the proof given in $[\mathrm{PD}]$ (Lemma 5.2.7) and refer to Section 5.6 in [PD] for the history of the theorem.

Theorem 1.3 If $\mathbb{Q} \subseteq A$ and $A=H^{\prime}(A)$, then for any $a \in A$

$$
a>0 \text { on } \operatorname{Sper} A \Longrightarrow a \in T \text {. }
$$

Proof. By the abstract Positivstellensatz (see e.g. III, §9, Theorem 4 in [KS]), we can choose $t \in T$ with $t a \in 1+T$. Since $A=H^{\prime}(A)$, there are $1 \leq \nu \in \mathbb{N}$ and $r \in \mathbb{N}$ such that $\nu-t \in T$ and $a+r \in T$. Now $r \in \frac{1}{\nu} \mathbb{N}$ can be made smaller and smaller until it is zero because

$$
a+\left(r-\frac{1}{\nu}\right)=\frac{\nu}{\nu^{2}}((\nu-t)(a+r)+(t a-1)+r t)
$$

is again in $T$ provided $r \geq 0$.

Having in mind this theorem, the new insight given by Schmüdgen's theorem is that $H^{\prime}(A)$ is as large as possible (namely $A=H^{\prime}(A)$ ) if $A=H(A)$ and the ring that underlies $A$ is a f.g. $\mathbb{R}$-algebra. Roughly speaking, in this work we will show that $H^{\prime}(A)$ is "as large as possible" (see below) dropping the restrictive assumption that $A=H(A)$.

We shall see that in some situations this helps to overcome the drawback of Theorem 1.3, namely the strong assumption $A=H^{\prime}(A)$ : Suppose $a>0$ on Sper $A$ and we want to show that $a \in T$, but the theorem is not directly applicable because $A=H^{\prime}(A)$ is violated. Then we can still try to find a preordered subring $B$ of $A$ such that $B=H^{\prime}(B), a \in B$ and $a>0$ even on Sper $B$ in order to apply the theorem. The larger we choose $B$ with the property $B=H^{\prime}(B)$ the better the chances that $a \in B$ and $a>0$ on Sper $B$. But there is a largest such $B$, namely - as the reader easily checks - $H^{\prime}(A)$. Hence the larger $H^{\prime}(A)$ the more likely Theorem 1.3 can be used to show $a \in T$.

To state precisely what we mean by $H^{\prime}(A)$ being "as large as possible", we define the $k$ times iterated ring of bounded elements $H^{k}(A)$ inductively by

$$
H^{0}(A):=A \quad \text { and } \quad H^{k+1}(A):=H\left(H^{k}(A)\right) \quad \text { for } k \in \mathbb{N} .
$$

One easily sees that

$$
H^{\prime}(A) \subseteq H^{k}(A) \quad \text { for every } k \in \mathbb{N} \text {. }
$$


Unfortunately, $H(A) \neq H^{2}(A)$ in general which prevents $H(A)=H^{\prime}(A)$, see the remarks after the proof of Theorem 3.11. However, the chain

$$
A \supseteq H(A) \supseteq H^{2}(A) \supseteq H^{3}(A) \supseteq \ldots
$$

of iterated rings of bounded elements stabilizes if (the ring underlying) $A$ is a f.g. $\mathbb{R}$-algebra. In fact, even if $A$ is an $\mathbb{R}$-algebra of finite transcendence degree $d \in \mathbb{N}$ (see Section 2 for this notion), then

$$
H^{d}(A)=H^{d+1}(A) .
$$

This was shown by Becker and Powers in $1996[\mathrm{BP}]$ for the case $T=\sum A^{2}:=$ $\left\{a_{1}^{2}+\cdots+a_{n}^{2} \mid n \in \mathbb{N}, a_{1}, \ldots, a_{n} \in A\right\}$. In Section 3 , we will give a new and comparatively short proof for the general case.

In Section 4, we will prove our main result: If $A$ is an $\mathbb{R}$-algebra of finite transcendence degree $d \in \mathbb{N}$, then

$$
H^{d}(A)=H^{\prime}(A) .
$$

In view of (1.2), this means that $H^{\prime}(A)$ is as large as one could have hoped for. This result was conjectured in 1998 by Monnier [Mon] and generalizes both the result of Schmüdgen and the result of Becker and Powers at the same time.

The proof is by induction on $d$. To make this induction work, we need, roughly speaking, three ingredients: Firstly, by modifying Wörmann's proof of Schmüdgen's theorem, we prove a statement about f.g. $\mathbb{R}$-algebras $A$ which is slightly stronger than (1.1). Secondly, we approximate $\mathbb{R}$-algebras of finite transcendence degree suitably by f.g. $\mathbb{R}$-algebras. This is necessary to handle the problem that (iterated) rings of bounded elements are in general far from being f.g. $\mathbb{R}$-algebras even if $A$ is so, see Remark 3.8. Finally, (1.3) enters the proof at several places.

Having proven our main result, we demonstrate how it helps to apply Theorem 1.3 in the way indicated above. Suppose $A$ is a (preordered) $\mathbb{R}$-algebra of finite transcendence degree (which needs not satisfy $A=H^{\prime}(A)$ ), $a \in A$ is what we call "small at infinity", i.e.

$$
\forall b \in A: \exists e \in \mathbb{N}: a^{e} b \in H(A),
$$

and $a \geq 0$ on Sper $A$. Using the chain

$$
A \supseteq H(A) \supseteq H^{2}(A) \supseteq \cdots \supseteq H^{d}(A)=H^{\prime}(A),
$$

we get hold of $H^{\prime}(A)$ and can show $a \in H^{\prime}(A)$ as well as $a+\varepsilon>0$ on $\operatorname{Sper} H^{\prime}(A)$ for each $\varepsilon>0$. Applying Theorem 1.3 to $H^{\prime}(A)$, we find $a+\varepsilon \in T$ for any $\varepsilon>0$.

In the literature, the ring of bounded elements is mostly called real holomorphy ring. This name which explains the notation $H(A)$ is motivated by the case where $A$ is a field furnished with a proper preordering $T$, which is mostly the set $\sum A^{2}$ of sums of squares, see the Notes on $\S 9$ in [Lam]. In this case, Becker, Schülting and others showed that $H(A)$ plays an important role 
in quadratic form theory, study of sums of $2 n$-th powers and algebraic geometry, see for example [Be1], [Be2], [Sc1], [Sc2], and III, §12 in [KS]. Some more recent works like [Ma1] partially extended this theory to the more general case where $1+T \subseteq A^{\times}$. In this case, $H(A)=H^{\prime}(A)$ (see $\S 11$, Korollar 1 in $[\mathrm{KS}]$ ) and most of our results become trivial. Becker and Powers [BP] initiated the study of the case $1+T \nsubseteq A^{\times}$. They were followed by Monnier, Pingel, Marshall and us who - motivated by Schmüdgen's theorem - also tackled the relationship between $H(A)$ and $H^{\prime}(A)$, see [Mon], [Pin] and [Ma2]. This theory differs much from the case $1+T \subseteq A^{\times}$and we followed Becker's suggestion to use another term instead of "real holomorphy ring."

Like our abstract above, a lot of articles only treat the case $T=\sum A^{2}$. Note that in this case $T_{H(A)}=\sum A^{2} \cap H(A)=\sum H(A)^{2}$, so that one stays within this setting when iterating rings of bounded elements. This has the advantage that one can simply work in the category of rings with which most readers are more familiar than with the category of preordered rings. It might be helpful to read some parts of the paper as if we worked in the former category. Nevertheless, we feel that it is more natural to use the latter one, not only because tricks like "adjoining roots" (compare the proof of Théorème 4.7 in [Mon]) can be avoided. For example, this allows for an interesting variant of our theory presented in Subsection 6.2, see Remark 6.1.

Acknowledgements The author thanks A. Prestel and N. Schwartz for drawing his attention to this interesting subject, E. Becker, S. and F.-V. Kuhlmann, M. Marshall for their warm hospitality during two one month stays in Dortmund and Saskatoon where part of this work was done and M. Aschenbrenner for proofreading an earlier version of this paper.

\section{Preliminaries}

For any set $\mathcal{M}$ of sets, we denote by $\mathcal{M}^{\text {min }}$ and $\mathcal{M}^{\text {max }}$ the subsets of its minimal and maximal elements, respectively.

We will need the notion of transcendence degree of a $K$-algebra $A$ where $K$ is a field. In the context of f.g. algebras, we will prefer to work with the then compatible Krull dimension which uses only the ring structure.

Definition 2.1 Let $K$ be a field and $A$ a $K$-algebra. In the following, we will take suprema of subsets of $\mathbb{N}$ in the set $\{-1\} \cup \mathbb{N} \cup\{\infty\}$ furnished with its natural ordering.

(i) Elements $a_{1}, \ldots, a_{n}$ of $A$ are called algebraically independent if there is no polynomial $0 \neq p \in K\left[X_{1}, \ldots, X_{n}\right]$ such that $p\left(a_{1}, \ldots, a_{n}\right)=0$ (in particular, the empty family of elements is algebraically independent unless $A=0)$.

(ii) The transcendence degree $\operatorname{trdeg} A$ of $A$ is the supremum of all $n \in \mathbb{N}$ such that there are $n$ algebraically independent elements in $A$ (thus $\operatorname{trdeg} A=$ $-1 \Longleftrightarrow A=0)$. 
(iii) The Krull dimension $\operatorname{dim} A$ of $A$ is the supremum of all $n \in \mathbb{N}$ such that there is a chain $\mathfrak{p}_{0} \subset \cdots \subset \mathfrak{p}_{n}$ of $n+1$ prime ideals $\mathfrak{p}_{0}, \ldots, \mathfrak{p}_{n} \in \operatorname{Spec} A$ (in particular, $\operatorname{dim} A=-1 \Longleftrightarrow A=0$ ).

We denote the (ordinary) transcendence degree of a field extension $L \mid K$ by $\operatorname{trdeg}(L \mid K) \in \mathbb{N} \cup\{\infty\}$. If $A$ is an integral domain, we write $\operatorname{qf}(A)$ for its quotient field.

Lemma 2.2 Let $K$ be a field and $A$ a $K$-algebra. Taking all suprema in the ordered set $\{-1\} \cup \mathbb{N} \cup\{\infty\}$, the following is true:

(i) $\operatorname{trdeg} A=\sup \{\operatorname{trdeg}(\operatorname{qf}(A / \mathfrak{p}) \mid K) \mid \mathfrak{p} \in \operatorname{Spec} A\}$

(ii) $\operatorname{trdeg} A=\sup \left\{\operatorname{trdeg}(\mathrm{qf}(A / \mathfrak{p}) \mid K) \mid \mathfrak{p} \in(\operatorname{Spec} A)^{\min }\right\}$

(iii) $\operatorname{dim} A \leq \operatorname{trdeg} A$

(iv) Af.g. $\Longrightarrow \operatorname{dim} A=\operatorname{trdeg} A<\infty$

(v) $B \subseteq A \Longrightarrow \operatorname{trdeg} B \leq \operatorname{trdeg} A$

(vi) If $A$ is an integral extension of $B$, then $\operatorname{trdeg} B=\operatorname{trdeg} A$.

(vii) If $\mathfrak{a}$ is an ideal of $A$, then $\operatorname{trdeg} A / \mathfrak{a} \leq \operatorname{trdeg} A$.

(viii) If $S \subseteq A$ is a multiplicative set, then $\operatorname{trdeg} S^{-1} A \leq \operatorname{trdeg} A$.

All these statements are proved in [Sim] except (ii). But the proof of (i) in [Sim] works verbatim for (ii).

A preordering $T$ of a ring $A$ is called archimedean if for each $a \in A$ there exists $\nu \in \mathbb{N}$ such that $\nu-a \in T$. According to this definition, for any preordered $\operatorname{ring} A, A=H^{\prime}(A)$ if and only if $T$ is archimedean.

\section{From now on $A$ and $B$ denote preordered rings.}

We call $\varphi: A \rightarrow B$ an homomorphism (embedding, isomorphism) if $\varphi$ is a ring homomorphism (embedding, isomorphism) and $\varphi\left(T_{A}\right) \subseteq T_{B}\left(\varphi\left(T_{A}\right)=T_{B} \cap\right.$ $\varphi(A))$. If $\mathfrak{a}$ is an ideal of $A$, then we furnish the $\operatorname{ring} A / \mathfrak{a}$ with the preordering $T_{A / \mathfrak{a}}:=\left\{a+\mathfrak{a} \mid a \in T_{A}\right\}$. If $S \subseteq A$ is a multiplicative set, then we equip the localization ring $S^{-1} A$ with the preordering $T_{S^{-1} A}:=\left\{\frac{a}{s^{2}} \mid a \in T_{A}, s \in S\right\}$. For any $a \in A$, we write $A_{a}$ for the localization $S^{-1} A$ of $A$ with respect to the multiplicative set $S:=\left\{1, a, a^{2}, \ldots\right\}$. Thus the preordering coming along with $A_{a}$ is $T_{A_{a}}=\left\{\frac{t}{a^{2 e}} \mid t \in T_{A}, e \in \mathbb{N}\right\}$. Note that $A_{0}=0$, i.e., $A_{0}$ is the preordered ring in which $0=1$.

Suppose $\mathcal{B}$ is a set of preordered subrings of $A$ which is directed, i.e., whenever $B_{1}, B_{2} \in \mathcal{B}$, there exists some $B \in \mathcal{B}$ with $B_{1} \subseteq B$ and $B_{2} \subseteq B$. If $A=\bigcup \mathcal{B}$, we say that $A$ is the directed union of all $B \in \mathcal{B}$. We will always express this in a sloppy way by writing

$$
\text { " } A=\bigcup_{\rightarrow} B, \quad \text { where } B \text { ranges over all elements of } \mathcal{B} . "
$$


Frequently, we will thereafter continue to speak about $B$ assuming tacitly that $B$ is an arbitrary element of $\mathcal{B}$.

By an algebra $A$, we will from now on always mean a preordered $K$-algebra $A$ where

- $K$ is a subfield of $\mathbb{R}$ and

- $K \subseteq H^{\prime}(A)$.

The latter condition ensures that $H(A), H(H(A)), \ldots$ and $H^{\prime}(A)$ are again algebras. It is fulfilled, for example, if every ordering of $K$ is archimedean or $\{a \in K \mid a \geq 0\} \subseteq T$. For example, regardless of $A$, we can always choose the field of rational, real algebraic or real numbers for $K$.

\section{$3 \quad$ Iterated rings of bounded elements}

In this section, we investigate the chain

$$
A \supseteq H(A) \supseteq H^{2}(A) \supseteq \ldots
$$

of iterated rings of bounded elements. We start with a simple observation.

Lemma 3.1 $H^{k}(A)$ is integrally closed in $A$ for each $k \in \mathbb{N}$.

Proof. By induction, it suffices to treat the case $k=1$. Suppose $a \in A$ and $b_{0}, \ldots, b_{n-1} \in H(A)$ satisfy the equation

$$
a^{n}+b_{n-1} a^{n-1}+\cdots+b_{1} a+b_{0}=0 .
$$

We must show that $a \in H(A)$. This follows from observing that for every $P \in \operatorname{Sper} A$

$$
|a(P)| \leq \max \left\{1,\left|b_{0}(P)\right|+\cdots+\left|b_{n-1}(P)\right|\right\} .
$$

Indeed, if $P \in \operatorname{Sper} A$ is such that $|a(P)| \geq 1$ then

$$
\begin{aligned}
|a(P)|^{n} & \leq\left|b_{n-1}(P)\right| \cdot|a(P)|^{n-1}+\cdots+\left|b_{0}(P)\right| \\
& \leq\left(\left|b_{0}(P)\right|+\cdots+\left|b_{n-1}(P)\right|\right) \cdot|a(P)|^{n-1} .
\end{aligned}
$$

Now divide both sides by $|a(P)|^{n-1}$.

Now we define some notions which will turn out to be very fruitful for the investigation of iterated rings of bounded elements. The respective terminology we suggest can be motivated, for instance, by Lemma 3.5, Proposition 3.6 and Corollary 3.7.

Definition 3.2 We define a subset $S_{\infty}(A)$ of $A$ by

$$
S_{\infty}(A):=\left\{a \in A \mid \forall b \in A: \exists e \in \mathbb{N}: a^{e} b \in H(A)\right\}
$$

and call its elements small at infinity. Next we define a subspace $\operatorname{Sper}_{\infty} A$ of the real spectrum of $A$ by

$$
\operatorname{Sper}_{\infty} A:=\{P \in \operatorname{Sper} A \mid P \text { is not archimedean }\} .
$$


The ideal

$$
I_{\infty}(A):=\bigcap\left\{P \cap-P \mid P \in \operatorname{Sper}_{\infty} A\right\}
$$

is the set of all $a \in A$ vanishing on $\operatorname{Sper}_{\infty} A$. We say that its elements vanish at infinity.

Since the non-archimedean orderings of $H(A)$ lie not in the image of the canonical map Sper $A \rightarrow$ Sper $H(A)$, any information we can obtain about them is valuable. Part (vi) of the following lemma provides us with such information: It says that every element of $S_{\infty}(A)$ vanishes at these orderings of $H(A)$.

Lemma 3.3 The following is true:

(i) $H(A)=\{a \in A \mid \forall P \in$ Sper $A: \exists \nu \in \mathbb{N}: \nu \pm a(P) \geq 0\}$

(ii) $H(A)=\left\{a \in A \mid \forall P \in \operatorname{Sper}_{\infty} A: \exists \nu \in \mathbb{N}: \nu \pm a(P) \geq 0\right\}$

(iii) $H(A)=\left\{a \in A \mid \exists \nu \in \mathbb{N}: \exists t \in T: t\left(\nu-a^{2}\right) \in 1+T\right\}$

(iv) $S_{\infty}(A)=\left\{a \in H(A) \mid H(A)_{a}=A_{a}\right\}$

(v) $I_{\infty}(A) \subseteq S_{\infty}(A)$

(vi) $S_{\infty}(A) \subseteq I_{\infty}(H(A))$

(vii) $I_{\infty}(A)$ is an ideal of $H^{k}(A)$ for each $k \in \mathbb{N}$.

(viii) $S_{\infty}(A)$ is an ideal of $H^{k}(A)$ for each $k \geq 1$.

Proof.

(i) We use that Sper $A$ is compact with respect to the constructible topology: Suppose $a \in A$ and for each $P \in \operatorname{Sper} A$ there is some $\nu_{P} \in \mathbb{N}$ such that $\nu_{P} \pm a(P) \geq 0$. Then consider the open covering

$$
\text { Sper } A=\bigcup_{P \in \operatorname{Sper} A}\left\{Q \in \operatorname{Sper} A \mid \nu_{P} \pm a(Q) \geq 0\right\}
$$

of Sper $A$ furnished with the constructible topology and extract a finite subcovering.

(ii) is a trivial consequence of (i).

(iii) The inclusion " $\supseteq$ " is obvious. For " $\subseteq$ " we use the (abstract) Positivstellensatz (see e.g. III, $\S 9$, Theorem 4 in $[\mathrm{KS}]$ ).

(iv) We first note that $S_{\infty}(A) \subseteq H(A)$. Indeed, for each $a \in S_{\infty}(A)$ there is some $e \in \mathbb{N}$ with $a^{e+1}=a^{e} \cdot a \in H(A)$ and thus $a \in H(A)$ by Lemma 3.1. Now fix some $a \in H(A)$. By the condition $H(A)_{a}=A_{a}$, we mean that the canonical homomorphism $\varphi: H(A)_{a} \rightarrow A_{a}$ is an isomorphism (of preordered rings). Since $\varphi$ always is an embedding this amounts to $\varphi$ being surjective. Obviously, this is equivalent to $a \in S_{\infty}(A)$.

(v) If $a \in I_{\infty}(A)$, then for all $b \in A$ we see that $a b \in H(A)$ using (ii). 
(vi) Let $a \in S_{\infty}(A)$ and $Q \in \operatorname{Sper}_{\infty} H(A)$. We have to show that $a(Q)=0$. Assume for a contradiction that $a(Q) \neq 0$. Then $Q$ comes from some ordering of $H(A)_{a}$. But according to (iv), $H(A)_{a}=A_{a}$ and the orderings of $A_{a}$ correspond to the orderings $P$ of $A$ with $a(P) \neq 0$. Thus there is some $P \in \operatorname{Sper} A$ with $Q=P \cap H(A)$. By definition of $H(A)$, the ordering $P \cap H(A)$ clearly is archimedean. This contradicts the hypothesis that $Q$ is not archimedean.

(vii) Combining (v) and (vi), we get $I_{\infty}(A) \subseteq I_{\infty}(H(A))$. By iteration, we see

$$
I_{\infty}(A) \subseteq I_{\infty}(H(A)) \subseteq I_{\infty}\left(H^{2}(A)\right) \subseteq \ldots \subseteq I_{\infty}\left(H^{k}(A)\right) \subseteq H^{k}(A)
$$

for each $k \in \mathbb{N}$. It suffices to show that $I_{\infty}(A)$ is an ideal of $A$. But this follows from the fact that by definition $I_{\infty}(A)$ is the intersection of supports of orderings of $A$ thus of ideals of $A$.

(viii) Combining (vi) and (v), we get $S_{\infty}(A) \subseteq S_{\infty}(H(A))$. By iteration, we see

$$
S_{\infty}(A) \subseteq S_{\infty}(H(A)) \subseteq S_{\infty}\left(H^{2}(A)\right) \subseteq \ldots \subseteq S_{\infty}\left(H^{k}(A)\right) \subseteq H^{k}(A)
$$

for each $k \in \mathbb{N}$. It is enough to show that $S_{\infty}(A)$ is an ideal of $H(A)$. From the definition of $S_{\infty}(A)$ it is obvious that $H(A) S_{\infty}(A) \subseteq H(A)$ and $0 \in S_{\infty}(A)$. It remains to show that $S_{\infty}(A)$ is additively closed. Suppose $a_{1}, a_{2} \in S_{\infty}(A)$ and let $b \in A$. Choose $e \in \mathbb{N}$ such that $a_{1}^{e} b, a_{2}^{e} b \in H(A)$. Then $\left(a_{1}+a_{2}\right)^{2 e} b=a_{1}^{2 e} b+2 e\left(a_{1}^{2 e-1} b\right) a_{2}+\cdots+a_{2}^{2 e} b \in H(A)$.

The following remark won't be needed in the sequel:

Remark 3.4 $I_{\infty}(A)$ is a real radical ideal of $A$ and a fortiori of $H^{k}(A)$ for every $k \in \mathbb{N}$. This follows from the fact that $I_{\infty}(A)$ is the intersection of supports of orderings of $A$ and thus of real prime ideals of $A$. Likewise, it is easy to show that $S_{\infty}(A)$ is a real radical ideal of $H(A)$ and a fortiori of $H^{k}(A)$ for every $k \geq 1$.

Lemma 3.5 If $A=K\left[x_{1}, \ldots, x_{n}\right]$ is a f.g. algebra, then the following statements hold true:

(i) $\operatorname{Sper}_{\infty} A=\left\{P \in \operatorname{Sper} A \mid \forall \nu \in \mathbb{N}: \sum_{i} x_{i}^{2}(P) \geq \nu\right\}$

(ii) $I_{\infty}(A)=\{f \in A \mid \exists \nu \in \mathbb{N}: \forall P \in \operatorname{Sper} A$ :

$$
\left.\sum_{i} x_{i}^{2}(P) \geq \nu \Longrightarrow f(P)=0\right\}
$$

(iii) $S_{\infty}(A)=\left\{f \in A \mid \exists e \in \mathbb{N}: f^{e} \sum_{i} x_{i}^{2} \in H(A)\right\}$

Proof. (i) is an easy exercise. The inclusion "つ" of (ii) is clear by (i) and the definition of $I_{\infty}(A)$. To show the reverse inclusion, suppose $f \in I_{\infty}(A)$. By (i), this means that

$$
\text { Sper } A=\{P \in \operatorname{Sper} A \mid f(P)=0\} \cup \bigcup_{\nu \in \mathbb{N}}\left\{P \in \operatorname{Sper} A \mid \sum_{i} x_{i}^{2}(P)<\nu\right\} \text {. }
$$


By compactness of Sper $A$ with respect to the constructible topology, we can extract from this covering of Sper $A$ a finite subcovering. To prove (iii), suppose $f \in A$ and

$$
\sum_{i} x_{i}^{2} \in C:=\left\{g \in A \mid \exists e \in \mathbb{N}: f^{e} g \in H(A)\right\} .
$$

Then it is easy to see that $f \in H(A)$, use for example (i) above and (ii) of Lemma 3.3. It follows that $C$ is a subalgebra of $A$. From $\sum_{i} x_{i}^{2} \in C$ we easily get $x_{i}^{2} \in C$ and then $x_{i} \in C$. Thus $C=A$ which means $f \in S_{\infty}(A)$.

The next proposition and its corollary provide handy characterizations of elements small at infinity in geometric situations. We will not need them for the theory itself but for some of its applications presented in Section 5.

Proposition 3.6 Suppose $A$ is a f.g. algebra whose preordering $T$ is also finitely generated. Then

$$
S_{\infty}(A)=\left\{f \in A\left|\forall P \in \operatorname{Sper}_{\infty} A: \forall k \in \mathbb{N}: k\right| f(P) \mid<1\right\} .
$$

Proof. The inclusion " $\subseteq$ " is clear by the preceding lemma. For the reverse, suppose $f \in A$ is an element of the right hand side of the equation. We may assume that $A$ equals $K\left[X_{1}, \ldots, X_{n}\right] /\left(g_{1}, \ldots, g_{l}\right)$ equipped with the preordering generated by the residues of finitely many polynomials $t_{1}, \ldots, t_{m}$. Then we can evaluate $f$ at points of the closed semialgebraic set

$$
S:=\left\{x \in \mathbb{R}^{n} \mid g_{1}(x)=0, \ldots, g_{l}(x)=0, t_{1}(x) \geq 0, \ldots, t_{m}(x) \geq 0\right\}
$$

which embeds densely in Sper $A$. If $S$ is bounded then $\operatorname{Sper}_{\infty} A=\emptyset$ by (i) of the lemma above whence $f \in A=S_{\infty}(A)$. In the non-trivial case, the semialgebraic set $\{\|x\| \mid x \in S\} \subseteq \mathbb{R}$ is unbounded and therefore contains the interval $\left[r_{0}, \infty[\right.$ for some $0 \leq r_{0} \in \mathbb{R}$. Now for every $r \in\left[r_{0}, \infty\left[\right.\right.$ let $x_{r} \in S \subseteq \mathbb{R}^{n}$ denote the lexicographically largest point where the continuous function

$$
\{x \in S \mid\|x\|=r\} \rightarrow \mathbb{R}: x \mapsto|f(x)|
$$

takes on its maximum. (Note that every non-empty compact subspace of $\mathbb{R}^{n}$ contains a lexicographically largest point.) Set

$$
P:=\left\{h \in A \mid \exists r_{1} \in\left[r_{0}, \infty\left[: \forall r \in\left[r_{1}, \infty\left[: h\left(x_{r}\right) \geq 0\right\} .\right.\right.\right.\right.
$$

Using the fact that for every $h \in A$ the set $\left\{r \in\left[r_{0}, \infty\left[\mid h\left(x_{r}\right) \geq 0\right\} \subseteq\right.\right.$ $\mathbb{R}$ is semialgebraic, it is routine to verify that $P \in \operatorname{Sper}_{\infty} A$. This implies $\frac{1}{k} \pm f \in P$ for every $0<k \in \mathbb{N}$ by supposition. Considering the function $F:\left[r_{0}, \infty\left[\rightarrow \mathbb{R}: r \mapsto\left|f\left(x_{r}\right)\right|\right.\right.$, this translates into $\lim _{r \rightarrow \infty} F(r)=0$. Moreover, since $F$ is semialgebraic, it is continuous except at finitely many points, see e.g. the Monotonicity Theorem in [Dri]. By increasing $r_{0}$, we may assume that $F$ is continuous. By a Lojasiewicz inequality (an obvious variant of Corollaire 2.6.7 in [BCR]), we obtain $e, c \in \mathbb{N}$ such that $F(r)^{e} \leq c \frac{1}{r^{2}}$ for all $r \in\left[r_{0}, \infty[\right.$. Consequently, $-c \leq f(x)^{e} \sum_{i} x_{i}^{2} \leq c$ for every $x \in S$ with $\|x\| \geq r_{0}$. Finally, the density of $S$ in Sper $A$ together with (iii) of Lemma 3.5 implies $f \in S_{\infty}(A)$. 
Corollary 3.7 Let $A$ be the polynomial ring $K\left[X_{1}, \ldots, X_{n}\right]$ together with a preordering $T$ generated by polynomials $t_{1}, \ldots, t_{m}$. Set

$$
S:=\left\{x \in \mathbb{R}^{n} \mid t_{1}(x) \geq 0, \ldots, t_{m}(x) \geq 0\right\} .
$$

Then

$$
S_{\infty}(A)=\{f \in A|\forall \varepsilon>0: \exists r \in \mathbb{N}: \forall x \in S:\|x\| \geq r \Longrightarrow| f(x) \mid<\varepsilon\} .
$$

Proof. The inclusion " $\subseteq$ " is clear by the trivial part of (iii) in Lemma 3.5. Conversely, suppose $f$ is contained in the set on the right hand side. We use the proposition above to show $f \in S_{\infty}(A)$. No matter how big $0<k \in \mathbb{N}$, we find $r \in \mathbb{N}$ such that $-\frac{1}{k}<f<\frac{1}{k}$ on the set $\{x \in S \mid\|x\| \geq r\}$. Then $-\frac{1}{k}<f<\frac{1}{k}$ holds even on the closure of this set with respect to the constructible topology on Sper $A$. This closure which is $\left\{P \in \operatorname{Sper} A \mid \sum_{i} X_{i}^{2}(P) \geq r^{2}\right\}$ contains $\operatorname{Sper}_{\infty} A$ by (i) of Lemma 3.5.

The next remark shows that often $H(A)$ is not noetherian even when $A$ has very nice properties (cf. Proposition 2.3 in $[\mathrm{BP}]$ ).

Remark 3.8 Suppose $1 \leq k \in \mathbb{N}$ and $I_{\infty}(A)$ is f.g. in $H^{k}(A)$. Then either $A=H(A)$ or $I_{\infty}(A)$ contains only zero divisors of $A$. To see this, suppose $b_{1} \in I_{\infty}(A)$ is not such a zero divisor. Write $I_{\infty}(A)=H^{k}(A) b_{1}+\cdots+H^{k}(A) b_{n}$ for some $b_{2}, \ldots, b_{n} \in H^{k}(A)$. Now let $a \in A$ be arbitrary. We will show that $a \in H^{k}(A)$. Denote by $b$ the row vector with the entries $b_{1}, \ldots, b_{n}$. Since $I_{\infty}(A)$ is an ideal of $A$, all entries of the vector $a b$ lie again in $I_{\infty}(A)$. Hence we can choose a quadratic $n \times n$ matrix $M$ over $H^{k}(A)$ such that $a b=M b$. This can equivalently be expressed as $(a I-M) b=0$ where $I$ denotes the identity matrix. By Cramer's rule, we get $b_{1} \operatorname{det}(a I-M)=0$ whence $\operatorname{det}(a I-M)=0$ since $b_{1}$ is no zero divisor. So $a$ is integral over $H^{k}(A)$ and therefore an element of $H^{k}(A)$ by Lemma 3.1 .

Lemma $3.9 H^{k}(A) / I_{\infty}(A)=H^{k}\left(A / I_{\infty}(A)\right)$ for every $k \in \mathbb{N}$

Proof. We proceed by induction on $k$. The case $k=0$ is trivial. We do the induction step from $k$ to $k+1$. For every $P \in \operatorname{Sper}_{\infty} H^{k}(A)$, we notice $I_{\infty}(A) \subseteq I_{\infty}\left(H^{k}(A)\right) \subseteq P \cap-P$. The homomorphism $H^{k}(A) \rightarrow H^{k}(A) / I_{\infty}(A)$ therefore induces a homeomorphism

$$
\operatorname{Sper}_{\infty} H^{k}(A) / I_{\infty}(A) \rightarrow \operatorname{Sper}_{\infty} H^{k}(A)
$$

and the following equalities hold:

$$
\begin{aligned}
H^{k+1} & (A) / I_{\infty}(A)=H\left(H^{k}(A)\right) / I_{\infty}(A) \\
& =\left\{a+I_{\infty}(A) \mid a \in H^{k}(A), \exists \nu \in \mathbb{N}: \nu \pm a \geq 0 \text { on } \operatorname{Sper}_{\infty} H^{k}(A)\right\} \\
& =\left\{b \in H^{k}(A) / I_{\infty}(A) \mid \exists \nu \in \mathbb{N}: \nu \pm b \geq 0 \text { on } \operatorname{Sper}_{\infty} H^{k}(A) / I_{\infty}(A)\right\} \\
& =H\left(H^{k}(A) / I_{\infty}(A)\right) \\
& =H\left(H^{k}\left(A / I_{\infty}(A)\right)\right) \quad \text { (by induction hypothesis) } \\
& =H^{k+1}\left(A / I_{\infty}(A)\right) \quad \square
\end{aligned}
$$


Lemma 3.10 If $\varphi: B \rightarrow A$ is a homomorphism (of preordered rings), then $\varphi(H(B)) \subseteq H(A)$. In particular, if $B$ is a preordered subring of $A$, then $H(B) \subseteq$ $H(A)$. Moreover,

$$
A=\bigcup_{\rightarrow} B \Longrightarrow H(A)=\bigcup_{\rightarrow} H(B)
$$

(where the $B$ of course are the same in both directed unions). This statement remains valid with $H$ replaced by $H^{\prime}$ everywhere.

Proof. The compatibility of $H$ with directed unions follows from (iii) of Lemma 3.3. The rest is straightforward.

The following theorem was discovered by Becker and Powers in 1996 for the case $T_{A}=\sum A^{2}$. Their proof uses concepts like regular points of algebraic sets which do not carry over to semialgebraic sets. However, by the technique of "adjoining roots", the applications can often be made fit into their framework. See for example the proofs of Théorème 4.7 and Théorème 5.12 in [Mon].

In 1998, two other works appeared, staying in the same setting than Becker and Powers: Monnier obtains additional information about the real spectra of $A, H(A), H^{2}(A), \ldots$, see Proposition 3.17 and Proposition 3.20 in [Mon]. Pingel sharpens under certain hypotheses the statement of the next theorem to $H^{d-1}(A)=H^{d}(A)$, see Satz 2.1.8 in [Pin].

Our proof is inspired by the proof of the theorem that in a field extension $E \mid F$ a chain of $d+1$ valuation rings $\mathcal{O}_{0} \subset \cdots \subset \mathcal{O}_{d}$ of $E$, all of which extend the same valuation $\operatorname{ring} \mathcal{O}$ of $F$, can only exist if $\operatorname{trdeg}(E \mid F) \geq d$, see Theorem A.6.7 in $[\mathrm{PD}]$.

Theorem 3.11 (Becker, Powers for $T=\sum A^{2}$ ) If $A$ is an algebra of finite transcendence degree $d \in \mathbb{N}$, then $H^{d}(A)=H^{d+1}(A)$.

Proof. We show by induction on $d \in \mathbb{N}$ that for every algebra $A$

$$
H^{d}(A) \supset H^{d+1}(A) \Longrightarrow \operatorname{trdeg} A \geq d+1
$$

(" $\supset$ " of course means proper inclusion). If $d=0$, then there is some $a \in$ $A \backslash H(A)$ which is not algebraic over $K$ by Lemma 3.1, so $\operatorname{trdeg} A \geq 1$. For the induction step, now suppose $d \geq 1$ and $H^{d}(A) \supset H^{d+1}(A)$. Write $A=\bigcup_{\rightarrow} B$ where $B$ ranges over all f.g. subalgebras of $A$. By iterated application of the preceding lemma, $H^{d}(A)=\bigcup_{\rightarrow} H^{d}(B)$ and thus $H^{d}(B) \supset H^{d+1}(B)$ for some f.g. subalgebra $B$ of $A$. Without loss of generality, we may therefore reduce to the case that $A$ is f.g., say $A=K\left[x_{1}, \ldots, x_{n}\right]$. By Lemma 3.9 , we have

$$
\begin{aligned}
H^{d-1}\left(H(A) / I_{\infty}(H(A))\right)= & H^{d}(A) / I_{\infty}(H(A)) \supset \\
& H^{d+1}(A) / I_{\infty}(H(A))=H^{d}\left(H(A) / I_{\infty}(H(A))\right)
\end{aligned}
$$

which implies $\operatorname{trdeg} H(A) / I_{\infty}(H(A)) \geq d$ by the induction hypothesis. Accordingly, we choose $a_{1}, \ldots, a_{d} \in H(A)$ whose residues are algebraically independent in $H(A) / I_{\infty}(H(A))$. We are going to show that $a_{1}, \ldots, a_{d}, \sum_{i} x_{i}^{2}$ are algebraically independent in $A$. So suppose $p_{0}, \ldots, p_{m} \in K\left[Y_{1}, \ldots, Y_{d}\right]$ are polynomials such that

$$
p_{m}(a)\left(\sum_{i} x_{i}^{2}\right)^{m}+p_{m-1}(a)\left(\sum_{i} x_{i}^{2}\right)^{m-1}+\cdots+p_{0}(a)=0
$$


where $a:=\left(a_{1}, \ldots, a_{d}\right)$. It is enough to show that $p_{m}=0$. This is clear if $m=0$. So assume $m>0$. Multiplying the above equation by $\left(p_{m}(a)\right)^{m-1}$, we see that $p_{m}(a) \sum_{i} x_{i}^{2}$ is integral over $H(A)$ and thus belongs to $H(A)$ by Lemma 3.1. This implies $p_{m}(a) \in S_{\infty}(A)$ by (iii) of Lemma 3.5. By (vi) of Lemma 3.3, $S_{\infty}(A) \subseteq I_{\infty}(H(A))$ which entails

$$
p_{m}\left(a_{1}+I_{\infty}(H(A)), \ldots, a_{d}+I_{\infty}(H(A))\right)=p_{m}(a)+I_{\infty}(H(A))=0
$$

in $H(A) / I_{\infty}(H(A))$. Then $p_{m}=0$ by the choice of $a$.

Pingel showed that the above theorem is sharp. She gave a lot of examples of f.g. algebras $A$ with

$$
\operatorname{trdeg} A>\operatorname{trdeg} H(A)>\cdots>\operatorname{trdeg} H^{d-1}(A)>\operatorname{trdeg} H^{d}(A),
$$

see Satz 2.3.7 in [Pin].

Recently Marshall found a very nice example of a f.g. algebra $A$ satisfying $H^{2}(A) \neq H(A)$ and $\operatorname{trdeg} H^{2}(A)=\operatorname{trdeg} A$, see Example 8.2 in [Ma5].

Note that the example at the end of $\S 4$ in $[\mathrm{BP}]$ and all its variations in [Pin] contain a subtle error: The last sentence in the proof of Claim 1 in $[\mathrm{BP}]$ is not true. Claim 1 is indeed wrong since $\frac{y(t-2)}{x} \in H(R) \backslash B[t]$. To show that $\frac{y(t-2)}{x} \notin B[t]$, one can use Gröbner bases. In fact, the $\mathbb{R}$-algebra $R$ in this example defines a variety whose (complex) points are all regular and which is therefore normal. Therefore Satz 2.1.8 in [Pin] tells us that $H(H(R))=H(R)$, i.e., the example fails.

\section{Generalizations of Schmüdgen's theorem}

In this section, $H^{\prime}(A)$ enters the picture. In case $\mathbb{Q} \subseteq A$, the following lemma will be generalized by Theorem 5.3 below.

Lemma 4.1 Suppose $\frac{1}{2} \in A$. Then for all $a \in A$

$$
a^{2} \in H^{\prime}(A) \Longrightarrow a \in H^{\prime}(A)
$$

Proof. For large $\nu \in \mathbb{N}$ we have $\left(\nu-\frac{1}{4}\right)-a^{2} \in T$ and hence

$$
\nu \pm a=\left(\nu-\frac{1}{4}\right)-a^{2}+\left(a \pm \frac{1}{2}\right)^{2} \in T .
$$

Lemma 4.2 If $A \supseteq B \supseteq H^{\prime}(A)$, then $H^{\prime}(A)=H^{\prime}(B)$. In particular,

$$
H^{\prime}(A)=H^{\prime}\left(H^{k}(A)\right) \quad \text { for each } k \in \mathbb{N} .
$$

Proof. By Lemma 3.10, we have $H^{\prime}(A) \supseteq H^{\prime}(B)$. To show $H^{\prime}(A) \subseteq H^{\prime}(B)$, suppose $a \in H^{\prime}(A)$, i.e., $\nu \pm a \in T$ for some $\nu \in \mathbb{N}$. Then $a \in H^{\prime}(A) \subseteq B$. Therefore $\nu \pm a \in T_{A} \cap B=T_{B}$ whence $a \in H^{\prime}(B)$. 
Lemma 4.3 Let $A=K\left[x_{1}, \ldots, x_{n}\right]$ be a f.g. algebra and $\varrho \in \mathbb{N}$. Then for every $f \in A$ there is some $\nu \in \mathbb{N}$ such that

$$
\nu-f \in(1+T)+(1+T)\left(\varrho-\sum_{i} x_{i}^{2}\right)=: \Sigma .
$$

Proof. If $f \in K$, we get some $\nu \in \mathbb{N}$ such that

$$
\nu \pm f \in\left(1+T+\sum_{i} x_{i}^{2}\right)+\left(\varrho-\sum_{i} x_{i}^{2}\right)
$$

from $K \subseteq H^{\prime}(A)$. If $f= \pm x_{i}$, it is enough to choose $\nu \geq \frac{5}{4}+\varrho$ because of the equation

$$
\left(\frac{5}{4}+\varrho\right) \pm x_{i}=1+\left(x_{i} \pm \frac{1}{2}\right)^{2}+\sum_{j \neq i} x_{j}^{2}+\left(\varrho-\sum_{i} x_{i}^{2}\right) .
$$

Now it suffices to show that the set of all $f \in A$ such that $\nu \pm f \in \Sigma$ for some $\nu \in \mathbb{N}$ is closed under addition and multiplication. Assume $f_{1}, f_{2} \in A$ and $\nu \pm f_{i} \in \Sigma$. Then

$$
2 \nu \pm\left(f_{1}+f_{2}\right) \in \Sigma+\Sigma \subseteq \Sigma
$$

and (observing that $\Sigma \Sigma \subseteq \Sigma$ )

$$
\nu^{2} \pm f_{1} f_{2}=\frac{1}{2}\left(\left(\nu \pm f_{1}\right)\left(\nu+f_{2}\right)+\left(\nu \mp f_{1}\right)\left(\nu-f_{2}\right)\right) \in \frac{1}{2}(\Sigma+\Sigma) \subseteq \Sigma .
$$

The following lemma is the key ingredient of our work. If $A=H(A)$, then $\operatorname{Sper}_{\infty} A=\emptyset$ whence $I_{\infty}(A)=A$. So in this case, the lemma is Schmüdgen's Positivstellensatz 1.1 minus Theorem 1.3. Our proof is much inspired by Wörmann's nice proof. One of the differences is that we (implicitly) use a Nullstellensatz instead of a Positivstellensatz.

Crucial Lemma 4.4 If $A$ is a f.g. algebra, then $I_{\infty}(A) \subseteq H^{\prime}(A)$.

Proof. Write $A=K\left[x_{1}, \ldots, x_{n}\right]$. Suppose $f \in I_{\infty}(A)$. According to (ii) of Lemma 3.5, we choose $\varrho \in \mathbb{N}$ such that $f(P)=0$ for all $P \in$ Sper $A$ with $\left(\sum_{i} x_{i}^{2}-\varrho\right)(P) \geq 0$. Of course,

$$
U:=T_{A_{f}}+\frac{\sum_{i} x_{i}^{2}-\varrho}{1} T_{A_{f}}
$$

is a preordering of $A_{f}$. If $U$ were a proper preordering, i.e., $-1 \notin U$, we could extend $U$ to an ordering of $A_{f}$. This would give rise to an ordering $P$ of $A$ with $\left(\sum_{i} x_{i}^{2}-\varrho\right)(P) \geq 0$ and $f(P) \neq 0$ contradicting the choice of $\varrho$.

Hence $-1 \in U$. Going back to $A$, we see that there exists $1 \leq e \in \mathbb{N}$ such that

$$
-f^{2^{e}} \in T+\left(\sum_{i} x_{i}^{2}-\varrho\right) T
$$


i.e., there is $t \in T$ such that

$$
t\left(\varrho-\sum_{i} x_{i}^{2}\right) \in f^{2^{e}}+T .
$$

Define $\Sigma$ like in Lemma 4.3. Then it follows that

$$
t \Sigma \subseteq f^{2^{e}}+T .
$$

Thus by Lemma 4.3, there exists for every $g \in A$ some $\nu \in \mathbb{N}$ such that $t(\nu-g) \in$ $f^{2^{e}}+T$. In particular, we find some $\nu \in \mathbb{N}$ such that

$$
t(\nu-t) \in f^{2^{e}}+T .
$$

Noting $t(\nu-t)=\frac{\nu^{2}}{4}-\left(t-\frac{\nu}{2}\right)^{2}$, we get

$$
\frac{\nu^{2}}{4} \in f^{2^{e}}+T
$$

This implies $f^{2^{e}} \in H^{\prime}(A)$. After applying Lemma 4.1 several times, we see that $f \in H^{\prime}(A)$.

As soon as we will have proved Corollary 4.17 it will be clear that in the lemma above and its corollary below the condition on $A$ to be f.g. can be weakened to $\operatorname{trdeg} A<\infty$.

Corollary 4.5 If $A$ is a f.g. algebra, then $H^{\prime}\left(A / I_{\infty}(A)\right)=H^{\prime}(A) / I_{\infty}(A)$.

Proof. The inclusion from the right to the left is trivial. To show the other direction, suppose $f+I_{\infty}(A) \in H^{\prime}\left(A / I_{\infty}(A)\right)$. Then there exists $\nu \in \mathbb{N}$ and $g \in I_{\infty}(A)$ such that $\nu+f+g \in T$. By the preceding theorem, there is some $\mu \in \mathbb{N}$ such that $\mu-g \in T$. Then $(\mu+\nu)+f \in T$. The same can be done with $-f$ instead of $f$. Therefore $f \in H^{\prime}(A)$ and $f+I_{\infty}(A) \in H^{\prime}(A) / I_{\infty}(A)$.

Definition 4.6 We call $A$ almost archimedian if every $P \in \operatorname{Sper} A$ with $P \cap$ $-P \in(\operatorname{Spec} A)^{\min }$ is archimedean.

Definition 4.7 We call an algebra $A$ finitely flavoured (f.f.) if $A$ has only finitely many minimal prime ideals $\mathfrak{p}$ and for each such $\mathfrak{p}$ the quotient field of $A / \mathfrak{p}$ is finitely generated over $K$, i.e., $\mathrm{qf}(A / \mathfrak{p})=K\left(s_{1}, \ldots, s_{n}\right)$ for some $s_{1}, \ldots, s_{n} \in \operatorname{qf}(A / \mathfrak{p})$.

Note that every f.f. algebra $A$ has finite transcendence degree by (ii) of Lemma 2.2. The following is an easy exercise in ring theory (see e.g. [Bou], II, Prop. 16).

Remark 4.8 If $B \subseteq A$, then for every $\mathfrak{q} \in(\operatorname{Spec} B)^{\min }$ there is $\mathfrak{p} \in(\operatorname{Spec} A)^{\min }$ such that $\mathfrak{q}=\mathfrak{p} \cap B$.

Lemma 4.9 All finitely generated algebras are finitely flavoured. All subalgebras of finitely flavoured algebras are again finitely flavoured. 
Proof. The first statement is clear. Now consider a subalgebra $B$ of a f.f. algebra $A$. By the above remark, $B$ has at most as many minimal prime ideals as $A$ and therefore only finitely many. It remains to show that for every $\mathfrak{q} \in(\operatorname{Spec} B)^{\text {min }}$ the field $\mathrm{qf}(B / \mathfrak{q})$ is finitely generated over $K$. Choose, as above, a minimal prime ideal $\mathfrak{p}$ of $A$ such that $\mathfrak{q}=\mathfrak{p} \cap B$. Now $\mathrm{qf}(B / \mathfrak{q})$ can be understood as a subfield of $\mathrm{qf}(A / \mathfrak{p})$ which is finitely generated over $K$. But intermediate fields of f.g. field extensions are again f.g. over the ground field, see for instance Lemma 9.30 in $[\mathrm{FJ}]$.

Lemma 4.10 Suppose $A$ is f.f. and almost archimedian. Then

$$
A=\bigcup_{\rightarrow} B, \quad \text { where } B \text { ranges over f.g., almost archimedian algebras. }
$$

Proof. For each $\mathfrak{p} \in(\operatorname{Spec} A)^{\text {min }}$, we can choose finitely many $a_{i} \in A, b_{i} \in A \backslash \mathfrak{p}$ (depending on $\mathfrak{p}$ ) such that $\mathrm{qf}(A / \mathfrak{p})$ is generated as a field by the fractions $\frac{a_{i}+\mathfrak{p}}{b_{i}+\mathfrak{p}}$. Now collect all of the these $a_{i}, b_{i}$ belonging to some $\mathfrak{p} \in(\operatorname{Spec} A)^{\mathrm{min}}$. Since $(\operatorname{Spec} A)^{\mathrm{min}}$ is finite, this remains a finite set. Therefore $A=\bigcup_{\rightarrow} B$ where $B$ ranges over all f.g. subalgebras of $A$ containing all $a_{i}, b_{i}$ associated to some $\mathfrak{p} \in(\operatorname{Spec} A)^{\min }$.

Now we fix such a $B$. It remains to show that $B$ is almost archimedian. Fix an arbitrary $Q \in \operatorname{Sper} B$ such that $\mathfrak{q}:=Q \cap-Q \in(\operatorname{Spec} B)^{\mathrm{min}}$. We have to show that $Q$ is archimedean. There is $\mathfrak{p} \in(\operatorname{Spec} A)^{\text {min }}$ such that $\mathfrak{q}=\mathfrak{p} \cap B$ by Remark 4.8. Viewing $B / \mathfrak{q}$ as a (preordered) subalgebra of $A / \mathfrak{p}$, we get $\mathrm{qf}(A / \mathfrak{p})=\mathrm{qf}(B / \mathfrak{q})$ (as preordered fields) for $B$ contains all the $a_{i}, b_{i}$ belonging to $\mathfrak{p}$. Consequently, there is some $P \in \operatorname{Sper} A$ such that $Q=P \cap B$ and $P \cap-P=$ $\mathfrak{p} \in(\operatorname{Spec} A)^{\mathrm{min}}$. The ordering $P$ and a fortiori $Q$ must be archimedean because $A$ is almost archimedian.

Whenever $\mathfrak{p}_{1} \cap \cdots \cap \mathfrak{p}_{n} \subseteq \mathfrak{p}$ for prime ideals $\mathfrak{p}_{1}, \ldots, \mathfrak{p}_{n}, \mathfrak{p}$ of a commutative ring then $\mathfrak{p}_{i} \subseteq \mathfrak{p}$ for some $i \in\{1, \ldots, n\}$. The next lemma generalizes this well-known fact.

Lemma 4.11 Suppose $C \subseteq \operatorname{Spec} A$ is closed with respect to the constructible topology. If $\mathfrak{p} \in \operatorname{Spec} A$ and $\bigcap C \subseteq \mathfrak{p}$, then there exists some $\mathfrak{q} \in C$ with $\mathfrak{q} \subseteq \mathfrak{p}$.

Proof. If such $\mathfrak{q}$ does not exist, then

$$
\operatorname{Spec} A=((\operatorname{Spec} A) \backslash C) \cup \bigcup_{a \in A \backslash p} V(a)
$$

where $V(a)$ denotes the set of prime ideals containing $a$. By compactness of Spec $A$ equipped with the constructible topology, we get $a_{1}, \ldots, a_{n} \in A \backslash \mathfrak{p}$ such that $\operatorname{Spec} A=((\operatorname{Spec} A) \backslash C) \cup V\left(a_{1}\right) \cup \cdots \cup V\left(a_{n}\right)$. Then $a_{1} \cdots a_{n} \in(\bigcap C) \backslash \mathfrak{p}$.

Lemma 4.12 If $A$ is a f.g. algebra which is almost archimedian, then

$$
\operatorname{dim} A / I_{\infty}(A)<\operatorname{dim} A .
$$


Proof. Since $A$ is f.g., $\operatorname{Sper}_{\infty} A$ is closed in Sper $A$ (see (i) of Lemma 3.5) and therefore compact with respect to the constructible topology on Sper $A$. The mapping

$$
\text { supp : Sper } A \rightarrow \operatorname{Spec} A: P \mapsto P \cap-P
$$

is continuous with respect to the constructible topologies on both sides. Hence $C:=\operatorname{supp}\left(\operatorname{Sper}_{\infty} A\right) \subseteq \operatorname{Spec} A$ is compact and thus closed with respect to the constructible topology on $\operatorname{Spec} A$. Now assume for a contradiction that $I_{\infty}(A) \subseteq \mathfrak{p}$ for some $\mathfrak{p} \in(\operatorname{Spec} A)^{\min }$. Since $I_{\infty}(A)=\bigcap C$ and $\mathfrak{p}$ is minimal, it follows by Lemma 4.11 that in fact $\mathfrak{p} \in C$. This contradicts our assumption that $A$ is almost archimedian.

Theorem 4.13 If $A$ is an algebra of finite transcendence degree, then

$$
A=H(A) \Longrightarrow A=H^{\prime}(A) \text {. }
$$

Proof. We first restrict to the case where $A$ is a f.f. algebra. We proceed by induction on $d:=\operatorname{trdeg} A$. The case $d=-1$, i.e., $A=0$, is trivial. Now suppose $d \geq 0$ and $A=H(A)$. By Lemma 4.10, we can write

$$
A=\bigcup_{\rightarrow} B, \quad \text { where } B \text { ranges over f.g., almost archimedian algebras. }
$$

We want to apply the induction hypothesis to $H^{d}\left(B / I_{\infty}(B)\right)$. This is indeed possible: First, $H^{d}\left(B / I_{\infty}(B)\right)$ is a subalgebra of the f.g. algebra $B / I_{\infty}(B)$ and therefore f.f. by Lemma 4.9. Second, we have

$$
\begin{aligned}
\operatorname{trdeg} H^{d}\left(B / I_{\infty}(B)\right) \leq \operatorname{trdeg} B / I_{\infty}(B) & =\operatorname{dim} B / I_{\infty}(B) \\
& <\operatorname{dim} B=\operatorname{trdeg} B \leq \operatorname{trdeg} A=d
\end{aligned}
$$

where the strict inequality is provided by Lemma 4.12 because $B$ is f.g. and almost archimedian. Finally, Theorem 3.11 yields

$$
H^{d}\left(B / I_{\infty}(B)\right)=H^{d+1}\left(B / I_{\infty}(B)\right)
$$

(if $d \neq 0$ even $H^{d-1}\left(B / I_{\infty}(B)\right)=H^{d}\left(B / I_{\infty}(B)\right)$ ). Now applying the induction hypothesis, we get

$$
H^{d}\left(B / I_{\infty}(B)\right)=H^{\prime}\left(H^{d}\left(B / I_{\infty}(B)\right)\right)=H^{\prime}\left(B / I_{\infty}(B)\right)
$$

where the latter equality is provided by Lemma 4.2. We get $H^{d}\left(B / I_{\infty}(B)\right)=$ $H^{d}(B) / I_{\infty}(B)$ by Lemma 3.9 and (not forgetting that $B$ is f.g.) $H^{\prime}\left(B / I_{\infty}(B)\right)=$ $H^{\prime}(B) / I_{\infty}(B)$ by Corollary 4.5. Hence the above equation entails

$$
H^{d}(B)=H^{\prime}(B)
$$

as sets and therefore as algebras. Using Lemma 3.10, we get

$$
A=H^{d}(A)=H^{d}\left(\bigcup_{\rightarrow} B\right)=\bigcup_{\rightarrow} H^{d}(B)=\bigcup_{\rightarrow} H^{\prime}(B)=H^{\prime}\left(\bigcup_{\rightarrow} B\right)=H^{\prime}(A)
$$

which completes the induction step. Now the claim is proved for f.f. algebras, in particular for f.g. algebras. Now let $A$ be arbitrary with $d=\operatorname{trdeg} A<\infty$. Then

$$
A=\bigcup_{\rightarrow} B, \quad \text { where } B \text { ranges over all f.g. subalgebras of } A \text {. }
$$


For each $B$, we have $H^{d}(B)=H^{d+1}(B)$ by Theorem 3.11 and thus $H^{d}(B)=$ $H^{\prime}\left(H^{d}(B)\right)=H^{\prime}(B)$ by what we have just proved. As above, it follows that $A=H^{\prime}(A)$, using Lemma 3.10.

In [Ma2], Marshall shows that the above theorem fails badly when the assumption on the transcendence degree is omitted:

Example 4.14 (Marshall) For $n \in \mathbb{N}$, let $A_{n}$ be $\mathbb{R}\left[X_{1}, \ldots, X_{n}\right]$ equipped with the preordering generated by

$$
X_{1}, \ldots, X_{n},\left(1-X_{1}\right)\left(1+X_{2}\right),\left(1-X_{2}\right)\left(1+X_{3}\right), \ldots,\left(1-X_{n-1}\right)\left(1+X_{n}\right) .
$$

Then it is very easy to see that $H\left(A_{n+1}\right)=A_{n}$ for every $n \in \mathbb{N}$ as rings. To see that this equality holds as preordered rings is a bit harder (see Lemma 2 in [Ma2]). Now consider the preordered ring $A:=\bigcup_{n \in \mathbb{N}} A_{n}$. Then $H^{\prime}\left(A_{n}\right) \subseteq$ $H^{n}\left(A_{n}\right)=A_{0}=\mathbb{R}$ whence $H^{\prime}\left(A_{n}\right)=\mathbb{R}$ for all $n \in \mathbb{N}$. Now by Lemma 3.10,

$$
\begin{gathered}
H(A)=H\left(\bigcup_{n \in \mathbb{N}} A_{n}\right)=H\left(\bigcup_{n \in \mathbb{N}} A_{n+1}\right)=\bigcup_{n \in \mathbb{N}} H\left(A_{n+1}\right)=\bigcup_{n \in \mathbb{N}} A_{n}=A, \\
\text { but } H^{\prime}(A)=H^{\prime}\left(\bigcup_{n \in \mathbb{N}} A_{n}\right)=\bigcup_{n \in \mathbb{N}} H^{\prime}\left(A_{n}\right)=\bigcup_{n \in \mathbb{N}} \mathbb{R}=\mathbb{R} .
\end{gathered}
$$

Now we are able to prove several generalizations of Schmüdgen's theorem. The one we start with was conjectured by Monnier in [Mon].

Theorem 4.15 Let $A$ be an algebra of finite transcendence degree such that $A=H(A)$. Then for each $a \in A$

$$
a>0 \text { on } \operatorname{Sper} A \Longrightarrow a \in T \text {. }
$$

Proof. By Theorem 1.3, it suffices to show that $A=H^{\prime}(A)$. But this is exactly what the preceding theorem says.

The next theorem generalizes not only Schmüdgen's theorem but also the theorem of Becker and Powers 3.11. It was conjectured in [Mon] for $T=\sum A^{2}$.

Main Theorem 4.16 Let $A$ be an algebra such that $\operatorname{trdeg} A=d \in \mathbb{N}$. Then

$$
H^{d}(A)=H^{\prime}(A)
$$

Proof. By Theorem 3.11, $H^{d}(A)=H^{d+1}(A)$. Thus $H^{d}(A)=H^{\prime}\left(H^{d}(A)\right)=$ $H^{\prime}(A)$ by Theorem 4.13 and Lemma 4.2 .

C.Q.F.D.

Now we enrich this statement by what we have proved already in (v) and (vi) of Lemma 3.3.

Corollary 4.17 Suppose $\operatorname{trdeg} A=d \in \mathbb{N}$. Then

$$
\begin{aligned}
A \supseteq H(A) \supseteq H^{2}(A) \supseteq \cdots \supseteq H^{d}(A)= \\
H^{d+1}(A)=H^{\prime}(A)=S_{\infty}\left(H^{d}(A)\right)=I_{\infty}\left(H^{d}(A)\right) \supseteq \ldots \\
\quad \cdots \supseteq S_{\infty}(H(A)) \supseteq I_{\infty}(H(A)) \supseteq S_{\infty}(A) \supseteq I_{\infty}(A) .
\end{aligned}
$$


Consider a f.g. or any other algebra $A$ of finite transcendence degree. If $A \neq H(A)$, we cannot apply Theorem 1.3 directly to $A$ in order to show that some element $a \in A$ lies in the preordering $T$. However, if we are lucky, we can show that $a \in H^{\prime}(A)$ by the preceding theorem, and Theorem 1.3 can always be applied to $H^{\prime}(A)$ because $H^{\prime}(A)=H^{\prime}\left(H^{\prime}(A)\right)$ by Lemma 4.2. But then we have to check that even $a>0$ on Sper $H^{\prime}(A)$ instead of only $a>0$ on Sper $A$. According to circumstances, we can use the following theorem to get hold of Sper $H^{\prime}(A)$. The proof of Theorem 4.19 and Example 5.4 below demonstrate this method.

Theorem 4.18 Suppose $A$ is an algebra such that $\operatorname{trdeg} A<\infty$. Then

$$
S_{\infty}(A)=\left\{a \in A \mid H^{\prime}(A)_{a}=A_{a}\right\} .
$$

Proof. If $A=0$, there is nothing to show. So let $A \neq 0$ and $d:=\operatorname{trdeg} A \in \mathbb{N}$. If $a \in A$ is such that $H^{\prime}(A)_{a}=A_{a}$, then of course $H(A)_{a}=A_{a}$ whence $a \in S_{\infty}(A)$ by (iv) of Lemma 3.3. Conversely, let $a \in S_{\infty}(A)$. Then $a \in S_{\infty}\left(H^{k}(A)\right)$ for each $k \in \mathbb{N}$. Applying (iv) of Lemma 3.3 to this fact for every $k \in\{0, \ldots, d-1\}$, we obtain

$$
A_{a}=H(A)_{a}=H^{2}(A)_{a}=\cdots=H^{d}(A)_{a} .
$$

Now $H^{d}(A)=H^{\prime}(A)$ implies $H^{\prime}(A)_{a}=A_{a}$.

Theorem 4.19 Suppose $A$ is an algebra such that $\operatorname{trdeg} A<\infty$. Then for every $a \in S_{\infty}(A)$ such that $a \geq 0$ on Sper $A$ and for every $0 \neq \varepsilon \in T \cap K$ we have $a+\varepsilon \in T$.

Proof. By Corollary 4.17, $a \in S_{\infty}(A) \subseteq H^{\prime}(A)$. Thus $\frac{a}{1} \in H^{\prime}(A)_{a}$. By hypothesis,

$$
\frac{a}{1}>0 \text { on } \operatorname{Sper} A_{a} .
$$

The preceding theorem says $A_{a}=H^{\prime}(A)_{a}$. So we get

$$
\frac{a}{1}>0 \text { on } \operatorname{Sper} H^{\prime}(A)_{a}
$$

and thus

$$
a \geq 0 \text { on } \operatorname{Sper} H^{\prime}(A) .
$$

Adding $0 \neq \varepsilon \in T \cap K$ makes this inequality strict:

$$
a+\varepsilon>0 \text { on } \operatorname{Sper} H^{\prime}(A)
$$

Now we apply Theorem 1.3 to $H^{\prime}(A)$.

\section{Applications}

As a first application we generalize the main result in Marshall's paper [Ma4]. Marshall required $g \in 1+T$ whereas we only need $g \geq 1$ on $S$. Thus we could add a lot to the instances of the so called moment problem solved by Kuhlmann and Marshall, see Corollary 4.5 in [KM]. Moreover, we exchange Marshall's "absolute growth condition" by a relative one and provide information about the exponent $e$. 
Theorem 5.1 Let $A$ be the polynomial ring $K\left[X_{1}, \ldots, X_{n}\right]$ equipped with the preordering generated by the polynomials $t_{1}, \ldots, t_{m}$. Set

$$
S:=\left\{x \in \mathbb{R}^{n} \mid t_{1}(x) \geq 0, \ldots, t_{m}(x) \geq 0\right\} .
$$

Suppose $f, g \in A$ and $g \geq 1$ on $S$. Suppose $e \in \mathbb{N}$ is such that $g$ " "grows more rapidly" than $f$ on $S$, more exactly one of the following equivalent conditions is satisfied:

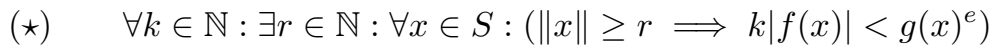

$(\star \star) \quad \forall P \in \operatorname{Sper}_{\infty} A: \forall k \in \mathbb{N}: k|f(P)|<g(P)^{e}$

$$
(\star \star \star) \quad \frac{f}{g^{e}} \in S_{\infty}\left(A_{g}\right)
$$

Then the following conditions are equivalent:

(i) $f \geq 0$ on $S$

(ii) For all $0<\varepsilon \in K$ there is some $k \in \mathbb{N}$ such that $g^{k}\left(f+\varepsilon g^{e}\right) \in T$.

Proof. From the density of $S$ in Sper $A$, we get $g \geq 1$ on Sper $A$. In particular, $g \neq 0$ on Sper $A$ which implies that Sper $A$ and Sper $A_{g}$ are canonically homeomorphic. Moreover, $\frac{1}{g} \in H\left(A_{g}\right)$ and therefore $\operatorname{Sper}_{\infty} A$ and $\operatorname{Sper}_{\infty} A_{g}$ are canonically homeomorphic (use (i) of Lemma 3.5). Now we get the equivalence of $(\star \star)$ and $(\star \star \star)$ by applying Proposition 3.6 to $A_{g}$. The implication $(\star \star \star) \Longrightarrow(\star)$ is trivial, and $(\star) \Longrightarrow(\star \star)$ can be proved analogously to Corollary 3.7. It is trivial that (ii) implies (i). Now suppose that $(\star \star \star)$ and (i) hold. Then Theorem 4.19 guarantees for arbitrary $0<\varepsilon \in K$

$$
\frac{f}{g^{e}}+\varepsilon \in T \text {. }
$$

A look at the definition of the preordering of $A_{g}$ yields (ii).

Remark 5.2 Sometimes condition $(\star \star)$ in the theorem above is easier to verify than $(\star)$. For example, it is easy to see that $(\star \star)$ always holds for $e=1$ and $g=1+f^{2} \sum_{i} X_{i}^{2}$ (a case which is not covered by [Ma4]) by distinguishing the cases that $f(P)$ is infinitesimaly close to zero, infinitesimaly close to a positive real number or infinitely large.

Next we give an example showing that the theory developed here might also be useful for the study of preordered rings which are not necessarily of finite transcendence degree over some field $K$. The following theorem can be found in Section 6.3 of $[\mathrm{Bru}]$ where a constructive proof is given.

Theorem 5.3 (Brumfiel) If $\mathbb{Q} \subseteq A$, then $H^{\prime}(A)$ is integrally closed in $A$.

Proof. Write $A=\bigcup_{\rightarrow} B$ where $B$ ranges over all f.g. $\mathbb{Q}$-subalgebras of $A$. Let $a \in A, b_{0}, \ldots, b_{n-1} \in H^{\prime}(A)$ be such that

$$
a^{n}+b_{n-1} a^{n-1}+\cdots+b_{1} a+b_{0}=0 .
$$


Since $H^{\prime}(A)=\bigcup_{\rightarrow} H^{\prime}(B)$, we can choose a f.g. $\mathbb{Q}$-subalgebra $B$ of $A$ such that

$$
b_{0}, \ldots, b_{n-1} \in H^{\prime}(B) \text { and } a \in B .
$$

Setting $d:=\operatorname{dim} B$, it follows by Lemma 3.1 that $H^{\prime}(B)=H^{d}(B)$ is integrally closed in $B$. Therefore $a \in H^{\prime}(B) \subseteq H^{\prime}(A)$.

The proof of Theorem 5.1 illustrated how easy it is to create concrete situations where Theorem 4.19 applies. If Theorem 4.19 cannot be applied, it is sometimes possible to argue in a customized way. This is illustrated in the next example.

Example 5.4 Let $A$ be the polynomial ring $\mathbb{Q}[X, Y]$ equipped with the preordering $T$ generated by $t_{1}:=1+(X+1)\left(1+Y^{2}\right), t_{2}:=1+(-X+1)\left(1+Y^{2}\right)$ and $t_{3}:=1+\left(X^{2}-1\right) Y^{2}$. We observe that $X+3 \geq 0$ on the set

$$
\begin{aligned}
S & :=\left\{(x, y) \in \mathbb{R}^{2} \mid t_{1}(x, y) \geq 0, t_{2}(x, y) \geq 0, t_{3}(x, y) \geq 0\right\} \\
& =\left\{(x, y) \in \mathbb{R}^{2}|| x \mid \leq 1+\frac{1}{1+y^{2}},\left(|x|<1 \Longrightarrow y^{2} \leq \frac{1}{1-x^{2}}\right)\right\} .
\end{aligned}
$$

This raises the question whether even $X+3 \in T$. By Corollary 3.7, it is easy to see that $X^{2}-1 \in S_{\infty}(A)$ (draw a picture of $S$ ). By Corollary 4.17, this implies $X^{2}-1 \in H^{\prime}(A)$ whence $X \in H^{\prime}(A)$ by the preceding theorem. Furthermore, $A_{X^{2}-1}=H^{\prime}(A)_{X^{2}-1}$ by Theorem 4.18. So for all $Q \in$ Sper $H^{\prime}(A)$ which are not in the image of Sper $A \rightarrow$ Sper $H^{\prime}(A)$, we have $X(Q) \in\{-1,1\}$. Together with $X+3>0$ on $S$ this shows $X+3>0$ on Sper $H^{\prime}(A)$. By Theorem 1.3, we get indeed $X+3 \in T$.

\section{Possible modifications of the theory}

In this section, we shortly describe possible variants of our theory. We don't go into details and refer the interested reader to $[\mathrm{Scw}]$.

\subsection{An alternative notion of vanishing at infinity}

We could replace $I_{\infty}(A)$ by

$$
I_{\infty}^{\prime}(A):=\bigcap\left\{P \cap-P \mid P \in\left(\operatorname{Sper}_{\infty} A\right)^{\max }\right\}
$$

in all the results stated so far without affecting their validity, except for (ii) of Lemma 3.5. This is interesting because $I_{\infty}^{\prime}(A)$ is the conductor of $A$ over $H(A)$, i.e., the largest ideal of $A$ which is contained in $H(A)$. If $T$ is f.g. in the f.g. algebra $A$, then also (ii) of Lemma 3.5 remains true in its modified form. To see this, one has to prove that $I_{\infty}(A)=I_{\infty}^{\prime}(A)$ in this case. This can be done, for instance, by using a Eojasiewicz inequality (in a way similar to the proof of Proposition 3.6, but the other way around).

But actually much more is true if $A$ and $T$ are finitely generated: In Sper $A$, the closure of the set of non-archimedean closed points is $\operatorname{Sper}_{\infty} A$ with respect to both the usual and the constructible topology. The latter fact can be proved using the ultrafilter theorem and techniques from semialgebraic geometry. 


\subsection{Quadratic modules and semiorderings}

The other possible modification is that we exchange preorderings and orderings by corresponding objects which are no more necessarily closed under multiplication but still closed under multiplication with squares. These objects were extensively used by Jacobi and Prestel to get "distinguished representations" of positive polynomials, see [JP], [PD] and [Ma3].

In lieu of preorderings we use quadratic modules which are subsets $T$ of a ring $A$ satisfying $0,1 \in T, T+T \subseteq T$ and $A^{2} T \subseteq T$. Every proper quadratic module (i.e., not containing -1 ) can be extended to a semiordering $P$ on $A$, i.e., a quadratic module $P$ of $A$ satisfying in addition $P \cup-P=A$ and $P \cap-P \in$ Spec $A$.

\section{Now let $A$ denote a ring equipped with a quadratic module $T$.}

The definition of $H^{\prime}(A)$ and the notion of archimedeaness extend canonically to this more general situation. We make the additional hypothesis $\frac{1}{2} \in A$ to show that $H^{\prime}(A)$ is still a ring: Since $H^{\prime}(A)$ clearly is a group with respect to addition and $a b=\left(\left(\frac{a+b}{2}\right)^{2}-\left(\frac{a-b}{2}\right)^{2}\right)$ for all $a, b \in A$, it is enough to show that $a \in H^{\prime}(A)$ implies $a^{2} \in H^{\prime}(A)$ for all $a \in A$. This can easily be derived from the fact that $\frac{1}{2} \pm a \in T$ implies

$$
\frac{1}{4}-a^{2}=\left(\frac{1}{2}+a\right)^{2}\left(\frac{1}{2}-a\right)+\left(\frac{1}{2}-a\right)^{2}\left(\frac{1}{2}+a\right) \in T .
$$

We write SemiSper $A$ for the space of all semiorderings of the ring underlying $A$ which contain the fixed quadratic module $T$ of $A$. Now we define

$$
\begin{aligned}
\operatorname{SemiH}(A) & :=\{a \in A \mid \exists \nu \in \mathbb{N}: \nu \pm a \geq 0 \text { on SemiSper } A\}, \\
\operatorname{SemiS}_{\infty}(A) & :=\left\{a \in A \mid \forall b \in A: \exists e \in \mathbb{N}: a^{e} b \in \operatorname{SemiH}(A)\right\}, \\
\operatorname{SemiSper}_{\infty} A & :=\{P \in \operatorname{SemiSper} A \mid P \text { is not archimedean }\} \text { and } \\
\operatorname{SemiI}_{\infty}(A) & :=\bigcap\left\{P \cap-P \mid P \in \operatorname{SemiSper}_{\infty}(A)\right\} .
\end{aligned}
$$

To see that $H(A)$ is a ring, note that $H(A)=H^{\prime}(B)$ where $B$ arises from $A$ by replacing the quadratic module $T_{A}$ by $T_{B}:=\bigcap\{P \mid P \in \operatorname{SemiSper} A\}$.

We will now outline how most of the theory carries over with all notions replaced by their equivalents just defined. Only the proofs of the more geometric theorems break down because SemiSper $A$ reflects not merely geometrical properties of $A$. Note that we deal here not with a generalization but with a variant of the theory: Though we aim to describe more general objects than before, the language we use to do this is now more obscure.

The most difficult point is to see that Theorem 1.3 remains valid in the new context. The tricky proof was achieved by Jacobi in 1999, see [Jac], Lemma 5.3.7 in [PD] or Theorem 5.1.4 in [Ma3]. As an exception to what we just said, Jacobi's theorem is really a generalization of Theorem 1.3. In fact, here it is not necessary to replace Sper $A$ by the bigger space SemiSper $A$. Theorem 1.3 can verbatim be stated like we $\operatorname{did}$ (where Sper $A$ is the space of orderings containing the quadratic module $T$ ). This is due to the favourable fact that

$$
\left(\operatorname{Sper} H^{\prime}(A)\right)^{\max }=\left(\operatorname{SemiSper} H^{\prime}(A)\right)^{\max },
$$


see Proposition 5.3.5 in [PD].

The proof of Lemma 3.1 now gets harder: From (i) in Lemma 3.3 it follows that

$$
H(A)=\left\{a \in A \mid \forall P \in \text { SemiSper } A: a(P) \in H^{\prime}\left(F_{P}\right)\right\}
$$

where $F_{P}:=\mathrm{qf}\left(B_{P} /(P \cap-P)\right)$ and $B_{P}$ arises from $A$ by replacing $T_{A}$ by $T_{B_{P}}:=P$. Now use the following facts: For every $P \in \operatorname{SemiSper} A, F_{P}$ is a semiordered field. Therefore $H^{\prime}\left(F_{P}\right)$ is a valuation ring of $F_{P}$ and a fortiori integrally closed in $F_{P}$, see Proposition 5.3.2 in [PD].

Next we have to replace (iii) of Lemma 3.3 by the following analogue supplied by the "weak Positivstellensatz" Theorem 5.1.10 in [PD]:

$$
\operatorname{SemiH}(A)=\left\{a \in A \mid \exists \nu \in \mathbb{N}: \exists \sigma \in \sum A^{2}: \sigma\left(\nu-a^{2}\right) \in 1+T\right\}
$$

Remark 6.1 If $T=\sum A^{2}$, then (iii) of Lemma 3.3 and its analogue describe the same set. Thus, in this case, $H(A)=\operatorname{SemiH}(A)$. Furthermore, since $T_{H(A)}=T \cap H(A)=\sum H(A)^{2}$, we see by the same argument that $H^{2}(A)=\operatorname{SemiH}^{2}(A)$ and inductively $H^{k}(A)=\operatorname{SemiH}^{k}(A)$ for $k \in \mathbb{N}$. Thus the described variant of our theory gives hardly new insight in the case $T=\sum A^{2}$.

To show (i) in Lemma 3.5, suppose $P$ is a semiordering of $A$ such that $\nu-\sum_{i} x_{i}^{2} \in P$. We have to show that $P$ is archimedean, in other words, $B=$ $H^{\prime}(B)$ where $B$ denotes the algebra arising from $A$ by replacing the preordering $T_{A}$ by $T_{B}:=P$. For every $i, x_{i}^{2} \in H^{\prime}(B)$ is immediate from $\nu-\sum_{i} x_{i}^{2} \in P$. Hence $x_{i} \in H^{\prime}(B)$ by Lemma 4.1. Therefore $B=H^{\prime}(B)$.

To be able to prove the Crucial Lemma 4.4, we make the additional hypothesis that $(T \cap K) T \subseteq T$ which is fulfilled for example if $K$ is the field of rational, real algebraic or real numbers. Since $(T \cap K)(T \cap K) \subseteq T$, the preordering generated by $T \cap K$ in the $\operatorname{ring} A$ is

$$
U:=\left\{\sum_{i=1}^{k} a_{i} f_{i}^{2} \mid k \in \mathbb{N}, f_{1}, \ldots, f_{k} \in A, a_{1}, \ldots, a_{k} \in T \cap K\right\} .
$$

Lemma 4.3 obviously even holds with $T$ replaced by $U$ and we have $U T \subseteq T$ since $(T \cap K) T \subseteq T$. We use this to show $t \Sigma \subseteq f^{2^{e}}+T$ in the proof of the Crucial Lemma.

All the results in our paper carry over to the different setting described above with the following exceptions: Theorem 1.1, Proposition 3.6, Corollary 3.7, Theorem 5.1, Example 5.4 and Subsection 6.1. Finally, we note that Jacobi's sharpening of Theorem 1.3 makes it unnecessary to replace Sper $A$ by SemiSper $A$ in Theorem 4.19:

Theorem 6.2 Suppose $A$ is an $\mathbb{R}$-algebra equipped with a quadratic module $T$ such that $\operatorname{trdeg} A<\infty$. Then for every $a \in \operatorname{SemiS}_{\infty}(A)$ such that $a \geq$ 0 on Sper $A$ and for every $0<\varepsilon \in \mathbb{R}$ we have $a+\varepsilon \in T$.

The proof is along the lines of Theorem 4.19. As a corollary, we obtain a new proof of Corollary 4.3 in [Ma5]. For simplicity, we restrict ourselves to a special case: 
Corollary 6.3 (Marshall) Let $A$ be $\mathbb{R}\left[X_{1}, \ldots, X_{n}\right]$ equipped with the quadratic module

$$
M:=\sum A^{2}+t_{1} \sum A^{2}+\cdots+t_{m} \sum A^{2}
$$

generated by $t_{1}, \ldots, t_{m} \in A$. Set

$$
S:=\left\{x \in \mathbb{R}^{n} \mid t_{1}(x) \geq 0, \ldots, t_{m}(x) \geq 0\right\} . \quad \text { and } \quad \sigma:=1+\sum_{i} X_{i}^{2} .
$$

Then for every $f \in A$ the following conditions are equivalent:

(i) $f \geq 0$ on $S$

(ii) $\exists l \in \mathbb{N}: \forall e \geq l: \forall 0<\varepsilon \in \mathbb{R}: \exists k \in \mathbb{N}: \sigma^{k}\left(f+\varepsilon \sigma^{e}\right) \in M$.

Proof. Clearly, (ii) implies (i). We now aim to show that $\frac{1}{\sigma} \in \operatorname{SemiS}_{\infty}\left(A_{\sigma}\right)$ using part (iii) of Lemma 3.5 (in its modified form). Since the $\mathbb{R}$-algebra $A_{\sigma}$ is generated by $\frac{X_{1}}{1}, \ldots, \frac{X_{n}}{1}$ and $\frac{1}{\sigma}$, it suffices to show that

$$
\frac{1}{\sigma}\left(\sum_{i} \frac{X_{i}^{2}}{1}+\frac{1}{\sigma^{2}}\right) \in \operatorname{SemiH}\left(A_{\sigma}\right)
$$

In fact, we will show that

$$
0 \leq \frac{1}{\sigma}\left(\sum_{i} \frac{X_{i}^{2}}{1}+\frac{1}{\sigma^{2}}\right) \leq 1 \quad \text { on SemiSper } A_{\sigma} .
$$

Since $\frac{1}{\sigma}=\frac{\sigma}{\sigma^{2}} \in \sum A^{2}$, it is enough to show that $0 \leq \sigma^{2} \sum_{i} X_{i}^{2}+1 \leq \sigma^{3}$ on SemiSper $A$. This is trivial.

Now suppose (i) holds for $f \in A$. Then even $f \geq 0$ on Sper $A$. Since $\frac{1}{\sigma} \in \operatorname{SemiS}_{\infty}\left(A_{\sigma}\right)$, we can find an $l \geq 1$ such that $\frac{f}{\sigma^{l-1}} \in \operatorname{SemiH}\left(A_{\sigma}\right)$ whence $\frac{f}{\sigma^{l}} \in \operatorname{SemiS}_{\infty}\left(A_{\sigma}\right)$. Then for every $e \geq l$, we have a fortiori $\frac{f}{\sigma^{e}} \in \operatorname{SemiS}_{\infty}\left(A_{\sigma}\right)$. Then for every $0<\varepsilon \in \mathbb{R}, \frac{f}{\sigma^{e}}+\varepsilon \in T$ by the previous theorem. Delocalizing now yields the result.

\section{References}

[BCR] J. Bochnak, M. Coste, M.-F. Roy: Géométrie algébrique réelle, Ergebnisse der Mathematik und ihrer Grenzgebiete 12, Berlin: SpringerVerlag (1987)

[Be1] E. Becker: The real holomorphy ring and sums of $2 n$-th powers, Géométrie algébrique réelle et formes quadratiques, Lect. Notes Math. 959, 139-181 (1982)

[Be2] E. Becker: Extended Artin-Schreier theory of fields, Rocky Mt. J. Math. 14, 881-897 (1984)

[Bou] N. Bourbaki: Commutative algebra, Paris: Hermann (1972)

[BP] E. Becker, V. Powers: Sums of powers in rings and the real holomorphy ring, J. Reine Angew. Math. 480, 71-103 (1996) 
[Bru] G. Brumfiel: Partially ordered rings and semi-algebraic geometry, London Mathematical Society Lecture Note Series 37, Cambridge: Cambridge University Press. (1979)

[BW] R. Berr, T. Wörmann: Positive polynomials on compact sets, Manuscr. Math. 104, 135-143 (2001)

[Dri] L. van den Dries: Tame topology and o-minimal structures, London Mathematical Society Lecture Note Series 248, Cambridge: Cambridge University Press (1998)

[FJ] M. Fried, M. Jarden: Field arithmetic, Ergebnisse der Mathematik und ihrer Grenzgebiete 11, Berlin: Springer-Verlag (1986)

[Jac] T. Jacobi: A representation theorem for certain partially ordered commutative rings, Math. Z. 237, 259-273 (2001)

[JP] T. Jacobi, A. Prestel: Distinguished representations of strictly positive polynomials, J. Reine Angew. Math. 532, 223-235 (2001)

[KM] S. Kuhlmann, M. Marshall: Positivity, sums of squares and the multidimensional moment problem, submitted

[KS] M. Knebusch, C. Scheiderer: Einführung in die reelle Algebra, Vieweg Studium 63, Braunschweig: Vieweg (1989)

[Lam] T. Lam: Orderings, valuations and quadratic forms, Reg. Conf. Ser. Math. 52 (1983)

[Ma1] M. Marshall: Orderings and real places on commutative rings, J. Algebra 140, 484-501 (1991)

[Ma2] M. Marshall: A real holomorphy ring without the Schmüdgen property, Can. Math. Bull. 42, 354-358 (1999)

[Ma3] M. Marshall: Positive Polynomials and Sums of Squares, Università di Pisa, Dipart. Mat., Istituti Editoriali e Poligrafici Internaz. (2000)

[Ma4] M. Marshall: Extending the Archimedean Positivstellensatz to the noncompact case, Can. Math. Bull. 44, 223-230 (2001)

[Ma5] M. Marshall: Approximating positive polynomials using sums of squares, submitted

[Mon] J.-P. Monnier: Anneaux d'holomorphie et Positivstellensatz archimédien, Manuscr. Math. 97, 269-302 (1998)

[PD] A. Prestel, C. Delzell: Positive polynomials, Springer Monographs in Mathematics, Berlin: Springer-Verlag (2001)

[Pin] S. Pingel: Der reelle Holomorphiering von Algebren, Dissertation, Fernuniversität Hagen (1998)

[Sc1] H.-W. Schülting: On real places of a field and their holomorphy ring, Commun. Algebra 10, 1239-1284 (1982) 
[Sc2] H.-W. Schülting: Real holomorphy rings in real algebraic geometry, Géométrie algébrique réelle et formes quadratiques, Lect. Notes Math. 959, 433-442 (1982)

[Sch] K. Schmüdgen: The $K$-moment problem for compact semi-algebraic sets, Math. Ann. 289, No.2, 203-206 (1991)

[Scw] M. Schweighofer: Iterated rings of bounded elements and generalizations of Schmüdgen's theorem, Dissertation, Universität Konstanz (2001)

[Sim] O. Simon: Aspects quantitatifs de Nullstellensätze et de Positivstellensätze, Nombres de Pythagore, Commun. Algebra 17, 637-667 (1989)

[Ste] G. Stengle: Complexity estimates for the Schmüdgen Positivstellensatz, J. Complexity 12, 167-174 (1996)

Universität Konstanz, Fachbereich Mathematik und Statistik

78457 Konstanz, Allemagne

schweigh@mathe uni-konstanz.de 